\title{
Phylogenetic analysis of porcine parvoviruses from swine samples in China
}

\author{
Xiaofang Hao, Zengjun Lu*, Pu Sun, Yuanfang Fu, Yimei Cao, Pinghua Li, Xingwen Bai, Huifang Bao, Baoxia Xie, \\ Yingli Chen, Dong Li and Zaixin Liu*
}

\begin{abstract}
Background: Porcine parvovirus (PPV) usually causes reproductive failure in sows. The objective of the present study was to analyze the phylogenetic distribution and perform molecular characterization of PPVs isolated in China, as well as to identify two field strains, LZ and JY. The data used in this study contained the available sequences for NS1 and VP2 from GenBank, as well as the two aforementioned Chinese strains.

Results: Phylogenetic analysis shows that the PPV sequences are divided into four groups. The early Chinese PPV isolates are Group I viruses, and nearly all of the later Chinese PPV isolates are Group II viruses. LZ belongs to group II, whereas the JY strain is a Group III virus. This is the first report on the isolation of a Group III virus in China. The detection of selective pressures on the PPV genome shows that the NS1 and VP2 genes are under purifying selection and positive selection, respectively. Moreover, the amino acids in the VP2 capsid are highly variable because of the positive selection.
\end{abstract}

Conclusions: Our study provides new molecular data on PPV strains in China, and emphasizes the importance of etiological studies of PPV in pigs.

\section{Background}

From a worldwide perspective, the porcine parvovirus (PPV) is one of the most common viral causes of porcine reproductive failure. PPV is of the genus Parvovirus, a group of viruses of that also infect cattle, cats, dogs, geese, rats, mice, mink, and raccoons. Although PPV is distinguishable from parvoviruses of all other species, it is antigenically related to other parvoviruses such as the canine parvovirus (CPV) and the feline panleukopenia virus (FPV) [1]. The PPV genome is a single strand DNA with a terminal palindromic structure. Its size is about $5 \mathrm{~kb}$. The PPV particle is composed of three viral polypeptides, VP1, VP2, and VP3, with molecular weights of 83,64 , and $62 \mathrm{kDa}$, respectively. In vitro expression of the VP2 gene may spontaneously form the capsid. The structure of the VP2 capsid was resolved using X-ray crystallography and was found to be similar to the CPV, FPV, and minute viruses of mice

\footnotetext{
* Correspondence: Iuzenjun920@126.com; liuzaixin3@hotmail.com
State Key Laboratory of Veterinary Etiological Biology, Key Laboratory of

* Correspondence: luzenjun920@126.com; liuzaixin3@hotmail.com
State Key Laboratory of Veterinary Etiological Biology, Key Laboratory of Animal Virology of Ministry of Agriculture, Lanzhou Veterinary Research
Institute, Chinese Academy of Agriculture Sciences, Lanzhou, Gansu 730046, Animal Virology of Ministry of Agriculture, Lanzhou Veterinary Research
Institute, Chinese Academy of Agriculture Sciences, Lanzhou, Gansu 730046, PR China
}

(c) 2011 Hao et al; licensee BioMed Central Ltd. This is an Open Access article distributed under the terms of the Creative Commons Attribution License (http://creativecommons.org/licenses/by/2.0), which permits unrestricted use, distribution, and reproduction in any medium, provided the original work is properly cited. cles mounted an immune response identical to that toward commercial vaccines [3]. Recently, the genome of PPV was found to contain a small open reading frame, designated as SAT, with a start codon downstream of the VP2 initiation codon [4]. In addition to these capsid proteins, some nonstructural polypeptides, such as NS1, NS2, and NS3, have also been identified in PPV-infected cells. Differentiation of infected pigs from vaccinated ones based on antibodies for NS1 protein using inactivated vaccine is possible, as NS1 protein is absent in purified virions used as a killed vaccine $[5,6]$.

Since the first discovery of PPV in 1983, the virus has spread widely in swine in China. Pigs are continuously threatened by PPV. Many new field strains were isolated from pigs in China, such as the China strain [6] and the BQ and ZJ strains [7]. Although inactivated and attenuated vaccines are widely used in swine in China, PPV infection is still a serious infectious disease. Therefore, porcine parvovirus surveillance in China is needed to learn the prevalence of PPV infections and to provide efficient epidemiological data for its control. 


\section{Results}

\section{Genomic sequences of the $L Z$ and JY strains}

The entire coding sequences of the LZ ad JY isolates contain 4509 and 4485 nucleotides, respectively, with no insertions or deletions in the coding regions. The sequences behind the VP2 stop codon of the LZ and JY isolates lack 105 and 127 nucleotides, respectively. This absence was also detected in some German isolates (e.g., PPV strains 15a, 143a, 106b, and Tornau), the American isolate Kresse, and some Chinese isolates (e.g., PPV strains BQ and ZJ). The complete sequences of LZ and JY strains were submitted to GenBank [GenBank: HM627652 and HM627653].

\section{Phylogenetic analysis of porcine parvoviruses isolated in China}

To characterize the genetic relationships of porcine parvoviruses isolated in China, and to identify the two PPV strains in this study, the phylogenetic trees of NS1 and VP2 nucleotide sequences were constructed based on the neighbor-joining method (Figure 1).

Four main groups or clades were formed in the NS1 and VP2 phylogenetic trees despite the fact that some isolates were not provided in the NS1 tree. Group I comprises some American strains (e.g., NADL-2, POVG, and Kresse), Chinese strains (e.g., China, SR-1, Tai'an, BQ, NJ, and N), the UK strain Challenge, and the Korean strain VRI-1. Group II contains the Chinese strains LZ and ZJ, some strains in the NS1 dataset (S-1, HN-Z1, Nanjing200801, Nanjng200802, NJ-2, HN-Z3, and Nanjing-1), and one strain in the VP2 dataset (LJL12). The strain SD-68 is placed in Group I in the NS1 tree, and under Group II in the VP2 tree. Group III contains three German isolates (21a, 27a, and 15a) and our isolate JY. Finally, Group IV is formed by five German isolates 225b, 143a, Tornau, 106b, and IDT. The Chinese isolates are mainly in Group I and II. Our isolate LZ belongs to Group II. However, another isolate, JY, was characterized as a Group III virus. This is the first isolation of a Group III virus in China.

\section{Selective pressure analysis of the PPV genome}

The selective pressures on the PPV genome were assessed by calculating the difference between non-synonymous $(\mathrm{dN})$ and synonymous $(\mathrm{dS})$ rates for the NS1 and VP2 genes. The average difference between $d N$ and $d S$ substitution rates $(\mathrm{dN}-\mathrm{dS})$ for NS1 was clearly negative
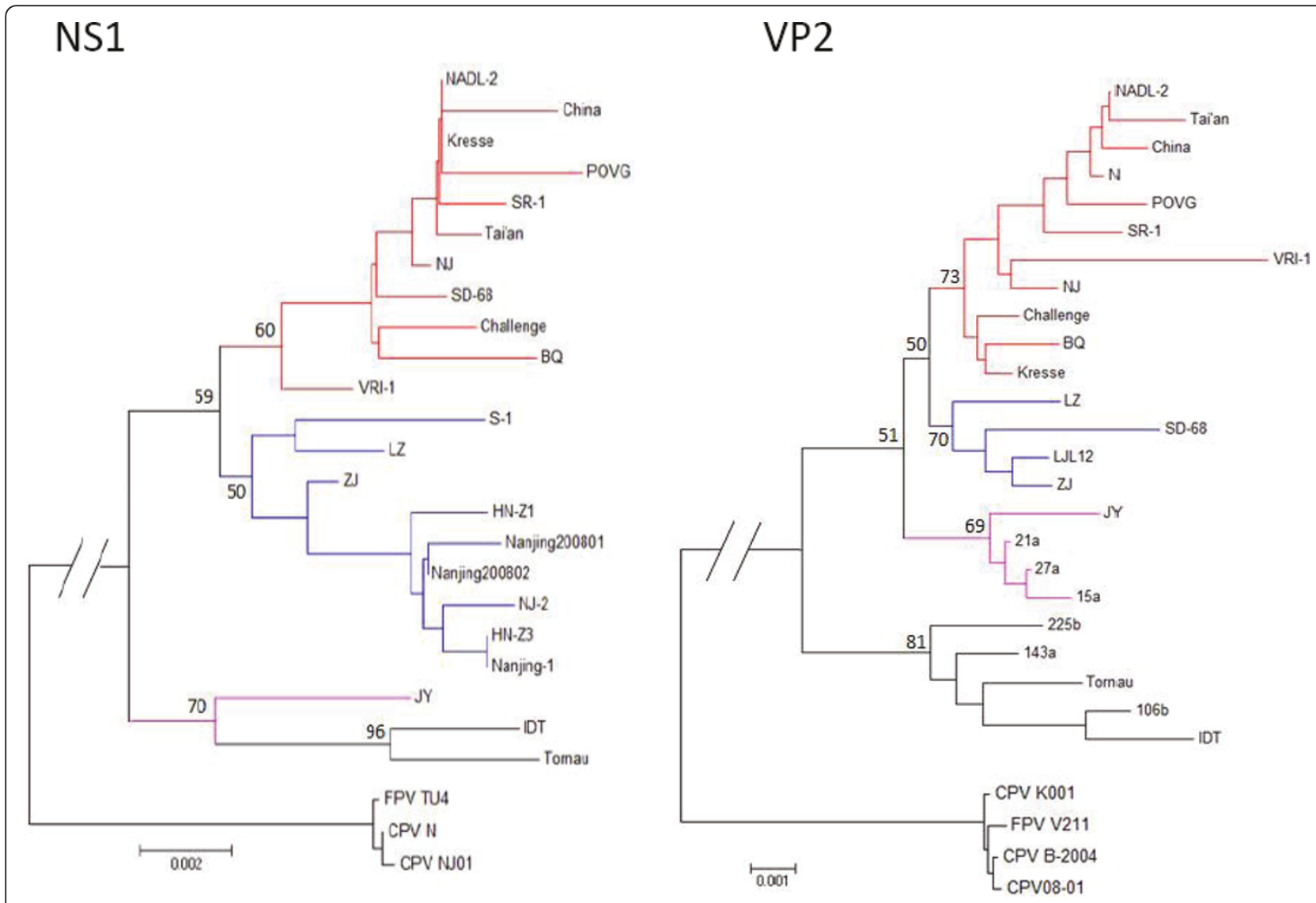

Figure 1 Phylogenetic trees based on the neighbor-joining method for the 23 NS1 and 24 VP2 sequences. The tree was constructed using MEGA version 4.1. Bootstrap values obtained from 1000 replicates are shown at the major nodes. The NS1 and VP2 sequences of CPV and FPV were included as outgroups. The different groups in the trees are marked by different colors. 
$(-0.009955 \pm 0.003446)$, based on the SNAP web utility http://hcv.lanl.gov/content/sequence/SNAP/SNAP.html results. This indicates that NS1 is under purifying selection. In contrast, the average $\mathrm{dN}-\mathrm{dS}$ for VP2 is clearly positive $(0.006535 \pm 0.003717)$, suggesting a positive selection pattern (Figure 2).

The distribution of $\mathrm{dN}-\mathrm{dS}$ along the NS1 sequence shows that the negative values are distributed among the first 130 amino acids and around residue 550 . According to previous studies, some amino acid residues of VP2 protein, such as $378,383,436$, and 565 , are crucial to the function of the VP2 capsid [2]. The $\mathrm{dN}-\mathrm{dS}$ at sites $378,383,436$, and 565 are $0.42,0.4,0.77$, and 0.33 , respectively, which indicates that all four residues are under positive selection.

\section{Molecular analysis of the VP2 capsid and amino acid} mutations in important residues

To find the molecular characteristics of the VP2 protein, the amino acid sequence alignments of the deduced VP2 coding sequences were constructed with representative strains aligned against the conserved sequence of all VP2 proteins. The alignment results of the complete VP2 proteins are presented in Figure 3. A number of polymorphic sites could be found along the VP2 amino acid sequence. Some mutations among the amino acids of the VP2 protein may distinguish different clades. For instance, Groups II and III viruses have a specific change at P436A and Q228E, respectively, whereas T20A, R82K, A93E, P304T, I320T, and K407N are unique to Group IV viruses.

In spite of the high levels of identity among the different parvovirus nucleotide sequences, parvovirus isolates can be distinguished by host range, cell tropism, and pathogenicity. In PPV, determinants for cell or tissue tropism, host range, and hemagglutination properties have been located within the capsid proteins. The biological significance of most of the sequence variations is unclear. However, some important amino acids in the VP2 protein have been identified by comparison of infectious clones of the NADL-2 and the Kresse strains $[8,9]$, and by structure elucidation of the PPV capsids

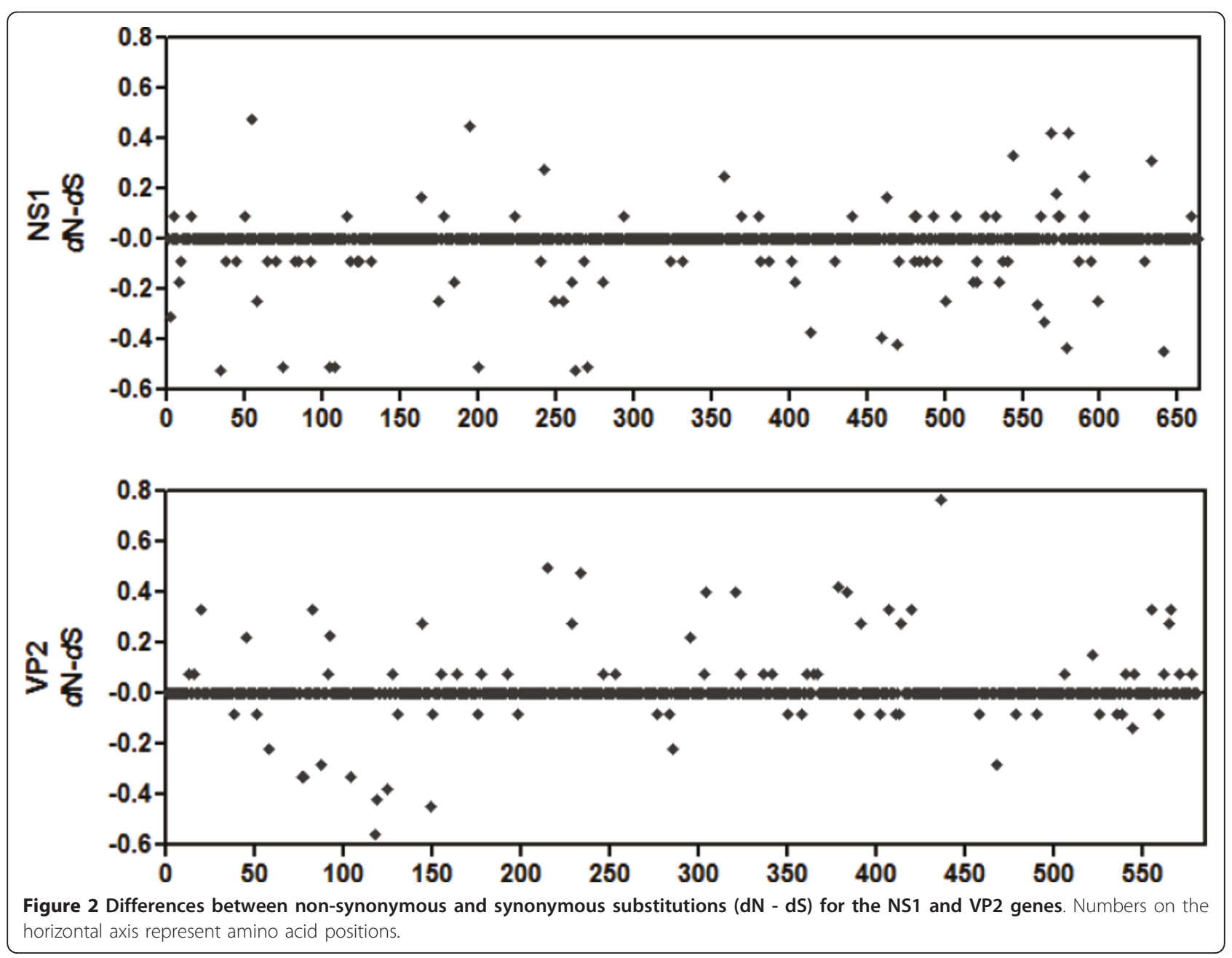




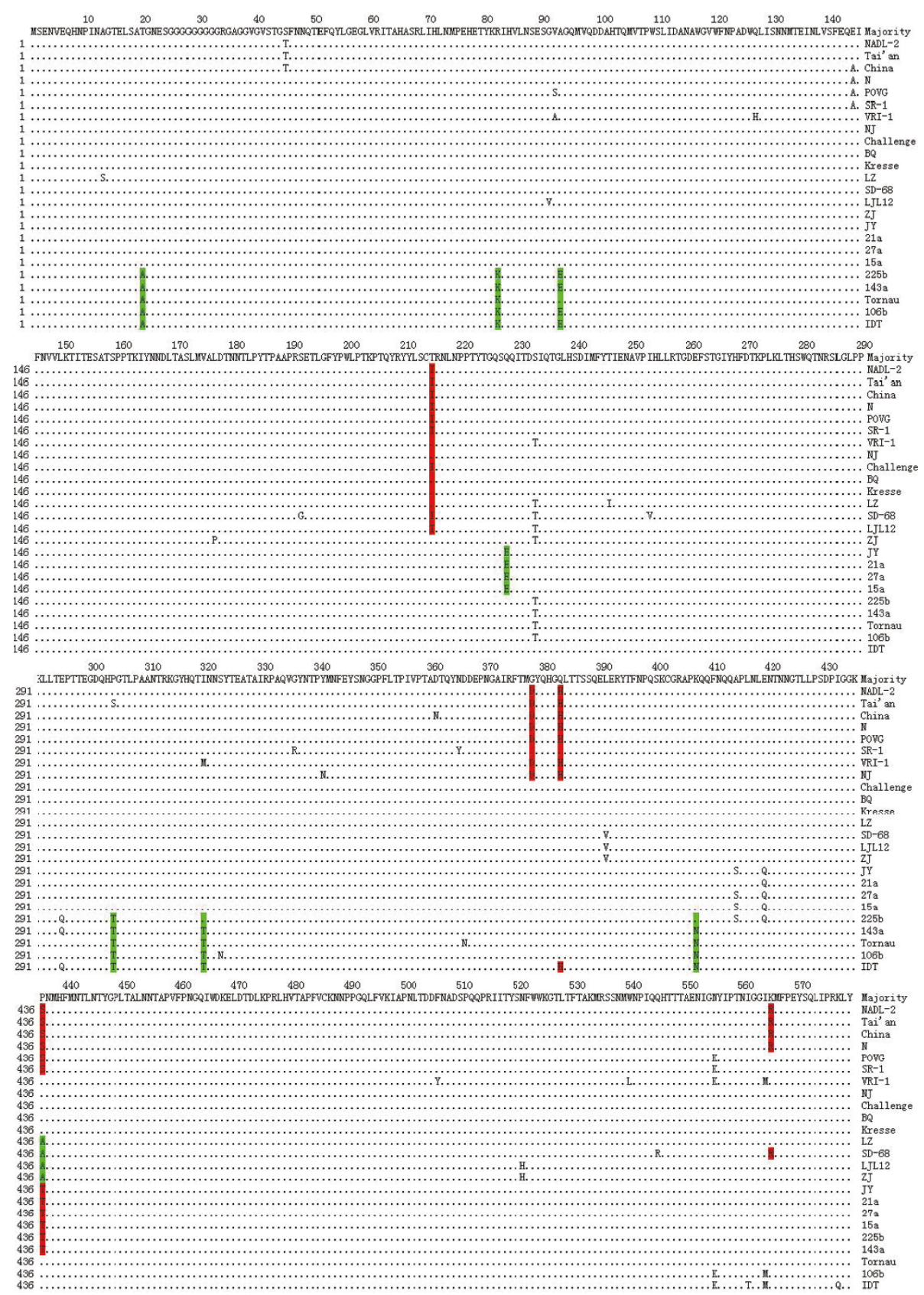

Figure 3 Sequence alignment of the deduced amino acid sequences of the VP2 gene. Important amino acid sites in the VP2 capsid are highlighted in different colors.

using X-ray crystallography [2]. For instance, the amino acids at positions 378,383 , and 436 are responsible for the allotropic determinant, as identified by the replacement of these residues with the Bgl II fragment in the infectious clone NADL-2. By mapping the key amino acid sites in the 3D structure of the capsid, some residues were found on the capsid surface. These residues are mapped to loop II for site 215, loops III and IV for sites 378 and 383, respectively, and to the C-terminal for site 565 . Residue 436 is located on top of the threefold spike within loop IV, which is the most accessible capsid structure and may be involved in tropism. Other residues, such as sites 314 and 509, are also important to the structural stability and biological function of the capsid because of their interaction with surface residues. As shown in Figure 3, the sequence difference mainly occurs at positions $215,378,383,436$, and 565 . The Chinese viruses of Group I have S436 or P436 residues. 
Residue A436 is unique to the Group II Chinese viruses. The Group III viruses and most of Group IV have the unique residue T436. The isolates JY and LZ have residues at T215, G378, Q383, and K565. However, a significant difference was found in A436 of the LZ strain and T436 of the JY strain.

\section{Sequence diversity of NS1 and VP2 protein}

The distribution of genetic diversity across the NS1 and VP2 proteins was investigated for 23 NS1 sequences and 25 VP2 sequences included in the previous phylogenetic analysis (Figure 4). The polymorphic diversity of the VP2 protein sequences was greater than that of NS1 protein sequences. The NS1 protein sequences contain $50 / 662(7.55 \%)$ polymorphic amino acid positions and have an overall amino acid diversity of $0.15 \pm 0.03$, whereas the VP2 protein sequences contain 48/579 (8.29\%) polymorphic amino acid positions and have an overall amino acid diversity of $0.26 \pm 0.05$.

\section{Discussion}

PPV is found in almost all pig-breeding countries. The PPV genome exhibits high genetic variation. Genetic analysis of the VP2 PPV gene in Brazilian isolates showed that two virus lineages existed in Brazilian swine populations [10]. Germany has a long PPV infection history. Hence, the genetic variability of German PPV isolates is complicated. Phylogenetic analysis of the fulllength VP1 nucleotide sequences of German isolates revealed two co-circulating clusters [11]. A further infection experiment showed that viruses of these two clusters are immunologically different, and indicated that the variation in PPV is not only at the gene level, but also at the antigenic level. Genome recombination in PPV has also been reported [12]. Conflicting phylogenetic histories of the different PPV genome regions provide evidence for recombination within these regions.

Various PPV strains have been isolated in field samples from China [6,7]. In the phylogenetic tree, the early Chinese PPV isolates, such as PPV strains N, China, and SR-1, are mainly located in Group I. Almost all later Chinese PPV isolates are Group II viruses, which are composed of Chinese strains, such as PPV strains ZJ, $\mathrm{NJ}-2$, and nanjing200801. The LZ isolate also belongs to group II in the NS1 and VP2 phylogenetic trees. JY, a Chinese PPV strain, falls under Group III, which is

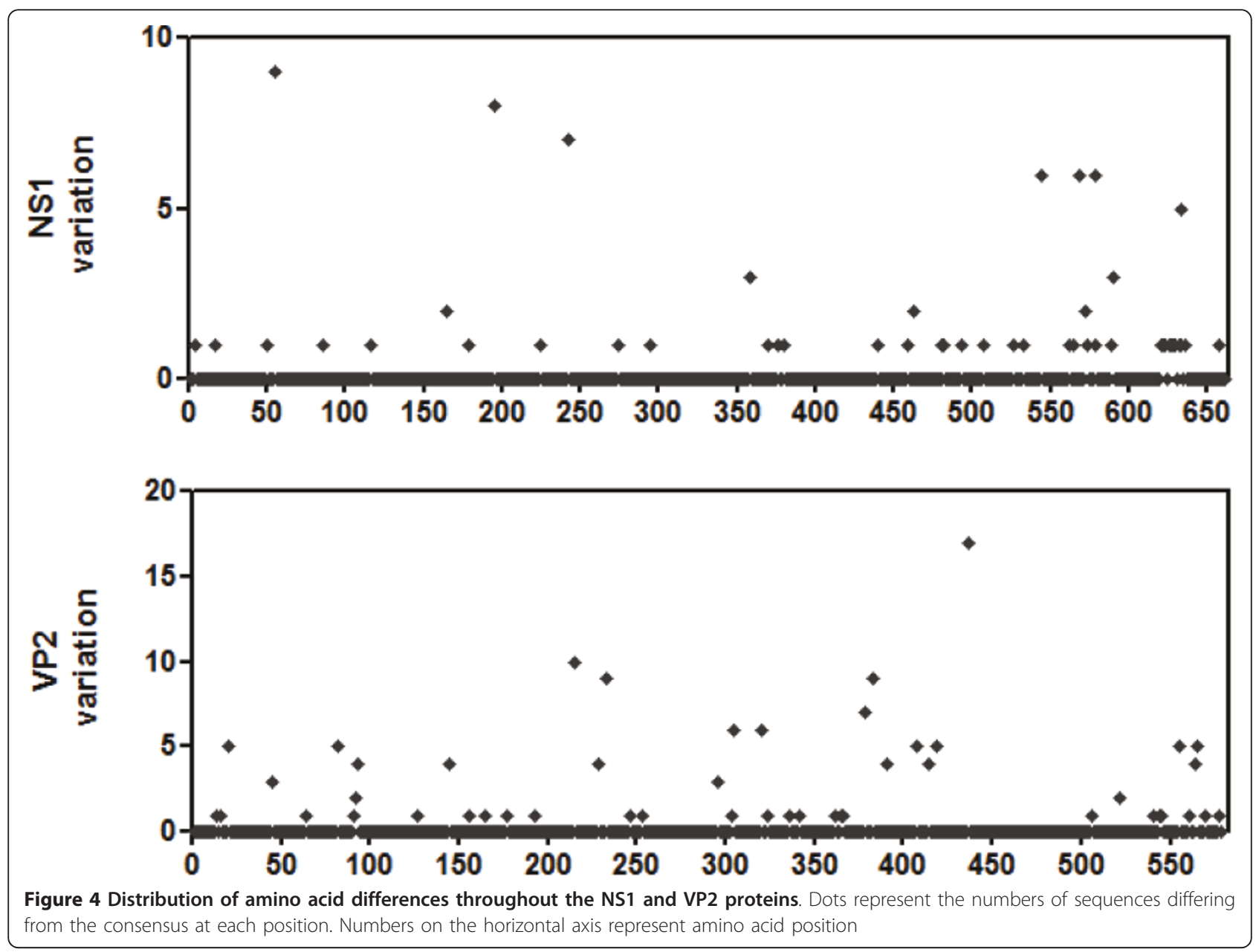


composed of German PPV isolates. This is the first isolation of a German-like PPV from field samples in China. This provides new molecular data on PPV strains in China, which may be used for etiological studies.

Considering the numerous PPV strains already sequenced, this study attempted to determine the genetic evolution of the PPV genome based on the selective pressures on the NS1 and VP2 genes. These PPV genes are under purifying selection and positive selection, respectively, as the average $\mathrm{dN}$ - dS for NS1 and VP2 genes are negative and positive, respectively. This suggests strong selection for the nonstructural proteins, which are less tolerant of amino acid alterations. The NS1 gene is more conserved than the VP2 gene. Hence, the NS1 gene may be regarded as the target gene for PPV detection. In contrast, the VP2 gene is inclined to mutate, especially in key points of the VP2 capsid, such as sites $378,383,436$, and 565 . These sites are all under positive selection, which indicates that the amino acid changes in these sites may be favorable for the survival of PPV.

The sequence lengths of the VP2 codon vary among different strains. The attenuated strain NADL-2 has a $127 \mathrm{bp}$ repeat, and it is considered to be distinct from the virulent isolates [8]. However, the repeat phenomenon was also found in the two virulent Chinese strains, China and SR-1. Hence, determining the virulence of a field strain based solely on the length of the repeat sequence is difficult. The JY strain lacks the $127 \mathrm{bp}$ repeat, similar to the field strains Kresse, 15a, Tornau, and 106b. The LZ strain, however, partially lacks nucleotides in the repeat region. Analogously, the lymphotropic variant of MVM, MVMi, also lacks a $65 \mathrm{bp}$ repeat [13]. The tandem repeat might be important for the replication of the viral genome because an infectious clone of MVMp, which lacks one copy of the repeat sequence, replicates at approximately $10 \%$ of wild viral levels in A9 cells. In addition, the repeats are very ATrich $(77 \%)$ and may have a negative effect on the stability of the transcripts [14].

\section{Conclusions}

The present study described the phylogenetic relationships, evolution, and genetic diversity of PPV strains isolated in China based on the NS1 and VP2 genes. A Group III virus from China was isolated for the first time. This study provides information for surveillance, prevention, and control strategies for PPV infections in China.

\section{Methods}

\section{Field samples}

From May 2009 to February 2010, swine in Gansu Province suffered from reproductive failure. Clinical samples, including lungs, kidneys, livers, and lymph nodes from pigs suffering from SMEDI (stillbirth, mummification, embryonic death, and infertility) were collected. Tissues samples from the pigs were homogenized for DNA extraction or virus isolation, and stored at $-70^{\circ} \mathrm{C}$. The sampling method was conducted in accordance with the guidelines on animal experimentation of Chinese academy of agricultural sciences (CAAS).

\section{Amplification of the of PPV whole sequences}

The viral genome was directly extracted from the homogenized tissues using the EZNA Tissue DNA kit (OMEGA, USA).

To understand the genetic characteristics of the newly isolated PPV strains, five pairs of primers were designed according to the conservative region of the whole sequence of PPV (Table 1). The amplified regions cover the whole genome of PPV with the exception of two terminal palindromic structures. The PCR reactions contained $4 \mu \mathrm{l}$ extracted DNA, $1 \mu \mathrm{l}$ primer pairs, $3 \mathrm{mM}$ dNTPs (TaKaRa, Dalian, China), $5 \mu \mathrm{l} 10 \times$ LA Taq buffer, and 0.5 U LA Taq polymerase (TaKaRa, Dalian, China) in a total volume of $50 \mu \mathrm{l}$. The PCR amplification was initiated by a pre-denaturation phage at $95^{\circ} \mathrm{C}$ for $5 \mathrm{~min}$, followed by 30 cycles of denaturation at $94^{\circ} \mathrm{C}$ for $1 \mathrm{~min}$, annealing at temperatures ranging from 54 to $59^{\circ} \mathrm{C}$ (Table 1) for $30 \mathrm{~s}$, and an extension at $72^{\circ} \mathrm{C}$ for 30-100 s (depending on the amplified fragment length). Subsequently, the PCR products were ligated into a pMD18-T vector (TaKaRa, Dalian, China) after gel extraction, and were used to transform Escherichia coli DH-5 $\alpha$-competent cells (TaKaRa, Dalian, China). The positive bacterial suspensions were sent to Invitrogen Corporation (Shanghai, China) for sequencing. The sequence assembly was carried out using the SeqMan program of the DNASTAR Software (Madison, WI).

\section{Sequence analysis}

Multiple sequence alignment was carried out using CLUSTAL W of the MegAlign program (DNASTAR), and the unrooted phylogenetic trees of NS1 and VP2 gene were generated by the neighbor-joining method using MEGA 4.1 software http://www.megasoftware.net/ mega4/mega41.html. Bootstrap values were calculated based on 1,000 replicates. Aside from the complete sequences of the two new Chinese strains JY and LZ, several genomic sequences were available from GenBank. All NS1 and VP2 genes available from GenBank were aligned to build the phylogenetic tree. The sequences of the VP2 proteins were also aligned to locate residue alterations. The virus isolates included 3 American strains, 8 Germany strains, 21 Chinese strains, 1 UK strain, and 1 South Korean strain (Table 2).

The selective pressures on the PPV genome were assessed by calculating the differences between the non- 
Table 1 Primers used in the amplification of the PPV genome

\begin{tabular}{|c|c|c|c|c|}
\hline Primer pairs & Location $^{\text {a }}$ & Sequences $\left(5^{\prime}-3^{\prime}\right)$ & Annealing temperature $\left({ }^{\circ} \mathrm{C}\right)$ & Amplicon length (bp) \\
\hline \multirow[t]{2}{*}{$\mathrm{A} 1$} & $244-1497$ & CACTTCGCTCCAGAGACACAGCTA & 58 & 1254 \\
\hline & & TGTTGATGCTGGCCCATGAAATAG & & \\
\hline \multirow[t]{2}{*}{$A 2$} & $1388-2356$ & TCAGCATGCACAATTGGAACTACA & 56 & 969 \\
\hline & & GTTTTATATGTATGCCCACCACCC & & \\
\hline \multirow[t]{2}{*}{ A3 } & 2214-3903 & GGAAATAGAAACCGACATAAGAGC & 55 & 1690 \\
\hline & & TTATATTGTGTGTCTGCTGTTGGT & & \\
\hline \multirow[t]{2}{*}{ A4 } & $3796-4456$ & AATTAGGCCAGCTCAGGTAGGATA & 59 & 661 \\
\hline & & TGTTGTTGTGTGTTGTTGAATAGG & & \\
\hline \multirow[t]{2}{*}{ A5 } & $4239-4854$ & GACTACATGTTACAGCTCCATTTG & 54 & $489-616 b$ \\
\hline & & ATAGTAAACACATGAGAGCTTGTT & & \\
\hline
\end{tabular}

\footnotetext{
a The position of the primer pairs are based on the entire genome sequence of the PPV NADL-2 strain [GenBank: NC_001718].

$\mathrm{b}$ The PCR products between 489-616 bp were all regarded as positive samples and sequenced, as a fragment deletion of 127 bp or less may occur in the amplification region of the $A 5$ primer pair.
}

Table 2 Details of the PPV isolates used in this study

\begin{tabular}{|c|c|c|c|c|}
\hline Isolate & $\begin{array}{l}\text { GenBank } \\
\text { accession no. }\end{array}$ & Origin & Dataset & Reference \\
\hline NADL-2 & NC 001718 & USA & NS1\&VP2 & [9] \\
\hline Kresse & U44978 & USA & NS1\&VP2 & [8] \\
\hline POVG & D00623 & USA & NS1\&VP2 & [17] \\
\hline Challenge & AY644866 & UK & NS1\&VP2 & [11] \\
\hline VRI-1 & AY390557 & $\begin{array}{l}\text { South } \\
\text { Korea }\end{array}$ & NS1\&VP2 & Unpublished \\
\hline 106b & AY684870 & Germany & VP2 & [11] \\
\hline $143 a$ & AY684867 & Germany & VP2 & [11] \\
\hline $15 a$ & AY684865 & Germany & VP2 & [11] \\
\hline $21 a$ & AY684868 & Germany & VP2 & [11] \\
\hline $225 b$ & AY684864 & Germany & VP2 & [11] \\
\hline $27 a$ & AY684871 & Germany & VP2 & [11] \\
\hline IDT & AY684872 & Germany & NS1\&VP2 & [11] \\
\hline Tornau & AY684869 & Germany & NS1\&VP2 & [11] \\
\hline $\mathrm{BQ}$ & EU790641 & China & NS1\&VP2 & [7] \\
\hline ZJ & EU790642 & China & NS1\&VP2 & [7] \\
\hline China & AY553318 & China & NS1\&VP2 & [6] \\
\hline $\mathrm{HN}-\mathrm{Z1}$ & AY789533 & China & NS1 & Unpublished \\
\hline $\mathrm{HN}-\mathrm{Z3}$ & AY789534 & China & NS1 & Unpublished \\
\hline LJL12 & DQ464345 & China & VP2 & Unpublished \\
\hline N & EF212027 & China & VP2 & Unpublished \\
\hline Nanjing-1 & AY739664 & China & NS1 & Unpublished \\
\hline Nanjing200801 & FJ822038 & China & NS1 & Unpublished \\
\hline Nanjing200802 & FJ822039 & China & NS1 & Unpublished \\
\hline NJ & AY686601 & China & NS1 & Unpublished \\
\hline NJ & AY686602 & China & VP2 & Unpublished \\
\hline NJ-2 & AY789532 & China & NS1 & Unpublished \\
\hline S-1 & EU707335 & China & NS1 & Unpublished \\
\hline SD-68 & AY502114 & China & NS1 & Unpublished \\
\hline SD-68 & AY502115 & China & VP2 & Unpublished \\
\hline SR-1 & DQ675456 & China & NS1/NP2 & Unpublished \\
\hline Tai'an & FJ853420 & China & NS1 & Unpublished \\
\hline Tai'an & FJ853421 & China & VP2 & Unpublished \\
\hline JY & HM627652 & China & NS1\&VP2 & This study \\
\hline LZ & HM627653 & China & NS1\&VP2 & This study \\
\hline
\end{tabular}

synonymous $(\mathrm{dN})$ and synonymous $(\mathrm{d} S)$ rates $(\mathrm{dN}-\mathrm{dS})$ for the NS1 and VP2 genes. The dN and dS rates were calculated using the SNAP web utility http://hcv.lanl. gov/content/sequence/SNAP/SNAP.html. SNAP calculates $\mathrm{dN}$ and $\mathrm{dS}$ rates for codon-aligned nucleotide sequences [15]. The ratios $d N-d S>0, d N-d S=0$, and $d N-d S<0$ signify positive selection (adaptive molecular evolution), neutral mutations, and negative selection (purifying selection), respectively [16].

\section{Acknowledgements}

This study was supported by the National High Technology Research and Development Program ("863" Program) of China (2011AA10A211) and a research grant from Gansu Province of China (GNSW-2009-10).

\section{Authors' contributions}

$Z J L$ and $Z X L$ designed the experiments; XFH, PS, YFF, YMC, PHL, XWB, HFB, $B X X, Y L C$, and DL performed the experiments and analyzed the data; $X F H$ and ZJL wrote the paper. All authors read and approved the final manuscript.

\section{Competing interests}

The authors declare that they have no competing interests.

Received: 18 March 2011 Accepted: 26 June 2011

Published: 26 June 2011

\section{References}

1. Mengeling WL, Ridpath JF, Vorwald AC: Size and antigenic comparisons among the structural proteins of selected autonomous parvoviruses. $J$ Gen Virol 1988, 69(Pt 4):825-837.

2. Simpson AA, Hebert B, Sullivan GM, Parrish CR, Zadori Z, Tijssen P, Rossmann MG: The structure of porcine parvovirus: comparison with related viruses. J Mol Biol 2002, 315(5):1189-1198.

3. Antonis AF, Bruschke CJ, Rueda P, Maranga L, Casal JI, Vela C, Hilgers LA, Belt PB, Weerdmeester K, Carrondo MJ, Langeveld JP: A novel recombinant virus-like particle vaccine for prevention of porcine parvovirus-induced reproductive failure. Vaccine 2006, 24(26):5481-5490.

4. Zadori Z, Szelei J, Tijssen P: SAT: a late NS protein of porcine parvovirus. J Virol 2005, 79(20):13129-13138

5. Qi T, Cui S: Expression of porcine parvovirus VP2 gene requires codon optimized E. coli cells. Virus Genes 2009.

6. Qing L, LV J, Li H, Tan Y, Hao H, Chen Z, Zhao J, Chen H: The recombinant nonstructural polyprotein NS1 of porcine parvovirus (PPV) as diagnostic antigen in ELISA to differentiate infected from vaccinated pigs. Vet Res Commun 2006, 30(2):175-190. 
7. Cui S, Mart J, Joaquim S: Phylogeny and evolution of the NS1 and VP1/ VP2 gene sequences from porcine parvovirus. Virus Research 2009, 140(12):209-215.

8. Bergeron J, Hebert B, Tijssen P: Genome organization of the Kresse strain of porcine parvovirus: identification of the allotropic determinant and comparison with those of NADL-2 and field isolates. J Virol 1996, 70(4):2508-2515.

9. Bergeron J, Menezes J, Tijssen P: Genomic organization and mapping of transcription and translation products of the NADL-2 strain of porcine parvovirus. Virology 1993, 197(1):86-98.

10. Soares RM, Cortez A, Heinemann MB, Sakamoto SM, Martins VG, Bacci M Jr, de Campos Fernandes FM, Richtzenhain L: Genetic variability of porcine parvovirus isolates revealed by analysis of partial sequences of the structural coding gene VP2. J Gen Virol 2003, 84(6):1505-1515.

11. Zimmermann P, Ritzmann M, Selbitz HJ, Heinritzi K, Truyen U: VP1 sequences of German porcine parvovirus isolates define two genetic lineages. J Gen Virol 2006, 87(Pt 2):295-301.

12. Shackelton LA, Hoelzer $K$, Parrish CR, Holmes EC: Comparative analysis reveals frequent recombination in the parvoviruses. J Gen Virol 2007, 88(Pt 12):3294-3301.

13. Astell CR, Gardiner EM, Tattersall P: DNA sequence of the lymphotropic variant of minute virus of mice, MVM(i), and comparison with the DNA sequence of the fibrotropic prototype strain. J Virol 1986, 57(2):656-669.

14. Sachs AB: Messenger RNA degradation in eukaryotes. Cell 1993, 74(3):413-421.

15. Nei M, Gojobori T: Simple methods for estimating the numbers of synonymous and nonsynonymous nucleotide substitutions. Mol Biol Evol 1986, 3:418-426.

16. Kosakovsky Pond SL, Frost SDW: Not So Different After All: A Comparison of Methods for Detecting Amino Acid Sites Under Selection. Molecular Biology and Evolution 2005, 22(5):1208-1222.

17. Ranz Al, Manclus JJ, Diaz-Aroca E, Casal Jl: Porcine parvovirus: DNA sequence and genome organization. J Gen Virol 1989, 70(Pt 10):2541-2553.

doi:10.1186/1743-422X-8-320

Cite this article as: Hao et al:: Phylogenetic analysis of porcine

parvoviruses from swine samples in China. Virology Journal 2011 8:320.

\section{Submit your next manuscript to BioMed Central and take full advantage of:}

- Convenient online submission

- Thorough peer review

- No space constraints or color figure charges

- Immediate publication on acceptance

- Inclusion in PubMed, CAS, Scopus and Google Scholar

- Research which is freely available for redistribution

Submit your manuscript at www.biomedcentral.com/submit

C BioMed Central 